\title{
Spatial polarimetric time-frequency distribution based DOA estimation: combining ESPRIT with MUSIC
}

\author{
Aijun Liu', Fen Li ${ }^{2}$, Bo Li $i^{*}$, Qingbo Liu² and Xiuhua Shi
}

\begin{abstract}
Spatial spectrum estimation technology has always been the focused research project in an array signal processing of High Frequency Surface Wave Radar (HFSWR). This paper takes the polarization sensitive array for the model and combines with time-frequency analysis method for DOA estimation of HFSWR echo signals, which can make full use of the spatial, time-frequency, and polarized domain information of signals. The time-frequency MUSIC and ESPRIT algorithms of integrated polarization information are studied, and the improved algorithm is proposed based on the advantages and disadvantages of both algorithms. The improved algorithm first estimates the azimuth angles of signals roughly with the polarized time-frequency ESPRIT algorithm and then determines a small scope centered on each azimuth. Within the scope, a more accurate DOA-estimated value is got by spectral peak search with MUSIC algorithm, so it can save computation time on the basis of accuracy of the DOA estimation, and their performance is analyzed by computer simulations.
\end{abstract}

Keywords: DOA estimation, Time-frequency, Polarization sensitive array

\section{Introduction}

In recent years, as the coastal area of space gets smaller to make HFSWR array antenna aperture get smaller too, using a signal processing technology to improve the precision of the direction of arrival (DOA) estimation algorithm has also become a research hotspot. The time-frequency analysis method uses the joint function of time and frequency to describe the energy density of the processed signal and has its unique advantage in dealing with nonstationary signals. In 1998, A. Belouchrani and M. Amin first observed that the Wigner-Ville distribution of the array output and the correlation matrix of the array output had a similar structure and applied it to the DOA estimation [1, 2]. Yimin Zhang [3, 4] found the intrinsic relation between the spatial time-frequency distribution matrix and the covariance matrix of the array output. For the first time, it has been proved theoretically that the structure of the spatial time-frequency distribution matrix can be used to estimate the DOA of the signals, which provides

\footnotetext{
* Correspondence: libo1983@hit.edu.cn

'School of Information and Electrical Engineering, Harbin Institute of

Technology (Weihai), Weihai, China

Full list of author information is available at the end of the article
}

theoretical support to study the time-frequency signal processing for later scholars. Polarization information is the inherent attribute of the electromagnetic wave signal, and it is also the information of the echo signals. The electromagnetic-vector-sensor (EMVS) has received extensive attention in an array signal processing due to the fact that it cannot only provide DOA of the signal, but can also give the polarization information. An electromagnetic vector-sensor usually consists of three orthogonally oriented dipoles to measure the electric field, plus three orthogonally oriented loops to measure the magnetic field of the source [5]. A unique DOA estimation algorithm for the EMVS, the vector-cross product algorithm, has been proposed in [5] and been advanced in [6, 7] along with various eigenstructure-based direction-finding schemes [8-10]. Dr. Ferrara and Parks of Stanford University in the USA used a polarized sensitive array composed of crossed dipole and combined with classical super resolution algorithms to study DOA [11]. Dr. Jian Li of the USA studied the polarization state parameters and the estimation of the DOA of electromagnetic waves in the case of polarized sensitive arrays based on uniform linear array [12]. In the literature [13], the polarization state parameters and DOA 
estimation in the case of polarized sensitive arrays based on rectangular arrays are studied. In [14], the joint estimation of the DOA and the polarization state in the case of the vector sensors in the absence of regularity is studied. In literature [15], the DOA and polarization parameters of the source signals are estimated firstly and then the variation of the estimated performance with the SNR is analyzed.

This paper intends to use the dual-polarized receiving antenna array from the compact high-frequency ground wave radar of the LFM system; make full use of the spatial, time-frequency, and polarized domain information of signals; and combined with the modern spatial spectrum estimation technologies. The super-resolution algorithm of time-frequency spectrum estimation of the integrated polarization information is studied.

\section{Signal model}

\subsection{Polarization sensitive array}

Polarization sensitive array refers to polarized sensitive elements arranged in a particular way in space, and the array can receive spatial and polarized domain information of the source signals. The spatial information can be expressed by the phase delay between different elements, but the polarization information needs to analyze the structure of the polarized sensitive elements. Polarization sensitive elements can receive six electromagnetic components most at the same time, but there will be some redundancy. By the relationship between the electric and magnetic field, a part of the components can be selected to form the element, but the polarization information of the electromagnetic wave signals can be obtained at least two components [16]. Therefore, it is usual to form a uniform linear array with orthogonal cross dipoles, as shown in Fig. 1. This structure is relatively simple and easy to achieve.

The polarization information of the electromagnetic wave signals can be expressed by the amplitude ratio and the phase difference of two mutually orthogonal electric fields, i.e.,

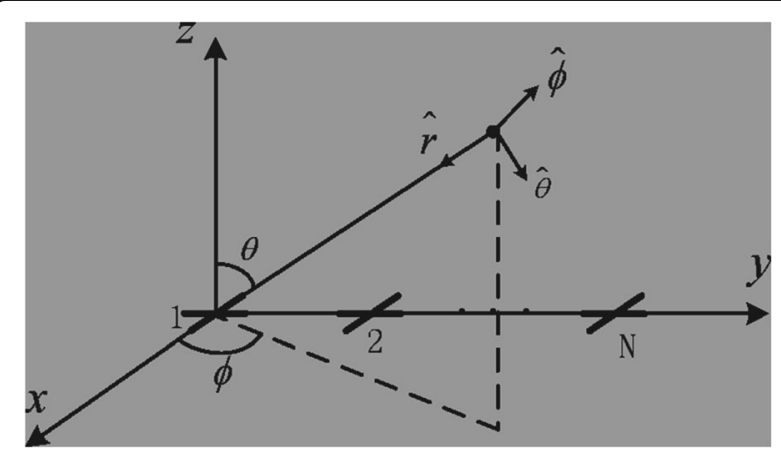

Fig. 1 Dual polarized antenna array

$$
\mathbf{e}=\left[\begin{array}{c}
A_{x} e^{j \phi_{x}} \\
A_{y} e^{j \phi_{y}}
\end{array}\right]=\left[\begin{array}{c}
\cos \gamma \\
\sin \gamma e^{j \eta}
\end{array}\right]
$$

where $\tan \gamma=\frac{A_{y}}{A_{x}}$ represents the ratio of electric field amplitudes between the $\mathrm{y}$-direction and the $\mathrm{x}$-direction, in addition, $\eta=\phi_{y}-\phi_{x}$ represents the phase difference of electric field between the $y$-direction and the $x$ direction. Regardless of the energy information of the electromagnetic wave, the polarization parameter $(\gamma, \eta)$ can represent the polarization information of any state.

When there are $k$ signal sources inciding $N$ elements array antenna and the noise is independent and stationary, the received signal can be expressed as

$$
\begin{aligned}
\mathbf{x}(t) & =\sqrt{N}\left[\begin{array}{llll}
\mathbf{s}_{1} & \mathbf{s}_{2} & \cdots & \mathbf{s}_{k}
\end{array}\right]\left[\begin{array}{c}
\mathbf{s}_{1}(t) \\
\mathbf{s}_{2}(t) \\
\vdots \\
\mathbf{s}_{k}(t)
\end{array}\right]+\mathbf{n}(t) \\
& =\sqrt{N} \mathbf{S} \cdot \mathbf{s}(t)+\mathbf{n}(t)
\end{aligned}
$$

where

$$
\mathbf{s}=\mathbf{s}_{\mathbf{p}} \otimes \mathbf{s}_{\mathbf{s}}
$$

represents the polarized-spatial domain joint steering vector of received signals, $S_{\mathrm{p}}$ is polarized domain steering vector, and $S_{\mathrm{s}}$ is spatial domain steering vector, symbol $\otimes$ represents kernel product of $S_{\mathrm{p}}$ and $S_{\mathrm{s}}$. The polarized domain steering vector can be expressed as

$$
\mathbf{s}_{\mathbf{p}}=\left[\begin{array}{l}
\mathbf{E}_{\mathbf{x}} \\
\mathbf{E}_{\mathbf{y}}
\end{array}\right]=\left[\begin{array}{cc}
-\sin \phi & \cos \theta \cos \phi \\
\cos \phi & \cos \theta \sin \phi
\end{array}\right]\left[\begin{array}{c}
\cos \gamma \\
\sin \gamma e^{j \eta}
\end{array}\right]
$$

and the spatial domain-oriented vector can be expressed as

$$
\mathbf{s}_{\mathbf{s}}=\frac{1}{\sqrt{N}}\left[\begin{array}{llll}
e^{j \phi_{1}} & e^{j \phi_{2}} & \cdots & e^{j \phi_{N}}
\end{array}\right]^{T}
$$

\subsection{Spatial polarimetric time-frequency distributions}

The Cohen's class of STFD of a data vector $x(t)$ is expressed as

$$
D_{x x}(t, f)=\iint \phi(t-u, \tau) x\left(u+\frac{\tau}{2}\right) x^{*}\left(u-\frac{\tau}{2}\right) e^{-j 2 \pi f t} d u d \tau
$$

where $\phi(t, \tau)$ is the time-frequency kernel function and it uniquely be used to define time-frequency distributions.

From the knowledge of time-frequency transform and the polarization sensitive array model introduced abovementioned, we can see that for the $k$ th dual polarized orthogonal dipole, the self-term and cross-term time-frequency distributions can respectively be expressed as 


$$
\mathbf{D}_{\mathbf{x}_{\mathbf{k}}^{[i]} \mathbf{x}_{\mathbf{k}}^{[i]}}(t, f)=\iint \phi(t-u, \tau) \mathbf{x}_{\mathbf{k}}^{[i]}\left(u+\frac{\tau}{2}\right)\left(\mathbf{x}_{\mathbf{k}}^{[i]}\left(u-\frac{\tau}{2}\right)\right)^{*} e^{-j 2 \pi f \tau} \boldsymbol{d} u \boldsymbol{d} \tau
$$

$$
\mathbf{D}_{\mathbf{x}_{\mathbf{k}}^{[i]} \mathbf{x}_{\mathbf{k}}^{[j}}(t, f)=\iint \phi(t-u, \tau) \mathbf{x}_{\mathbf{k}}^{[i]}\left(u+\frac{\tau}{2}\right)\left(\mathbf{x}_{\mathbf{k}}^{[j]}\left(u-\frac{\tau}{2}\right)\right)^{*} e^{-j 2 \pi f \tau} \boldsymbol{d} u \boldsymbol{d} \tau
$$

where the superscript $i$ and $j$ both represent the vertical component $v$ or the horizontal component $h$ of the array element.

Therefore, the time-frequency distribution of the $k$ th dual polarized orthogonal dipole is a $2 \times 2$ matrix, which consists of the vertical and horizontal oscillator's self-term and cross-term time-frequency distributions. Considering a uniform linear array consists of $n$ dual polarized orthogonal dipole, the received data vector can be expressed as

$$
\begin{aligned}
\mathbf{x}^{[i]}(t) & =\left[\mathbf{x}_{\mathbf{1}}^{[i]}(t), \mathbf{x}_{\mathbf{2}}^{[i]}(t), \cdots, \mathbf{x}_{\mathbf{N}}^{[i]}(t)\right]^{T} \\
& =\mathbf{y}^{[i]}(t)+\mathbf{n}^{[i]}(t)=\mathbf{A}^{[i]} \mathbf{s}^{[i]}(t)+\mathbf{n}^{[i]}(t)
\end{aligned}
$$

The time-frequency distributions of its self-term and cross-term can be expressed as

$\mathbf{D}_{\mathbf{x}^{[i]} \mathbf{x}^{[i]}}(t, f)=\iint \phi(t-u, \tau) \mathbf{x}^{[i]}\left(u+\frac{\tau}{2}\right)\left(\mathbf{x}^{[i]}\left(u-\frac{\tau}{2}\right)\right)^{H} e^{-j 2 \pi f \tau} \boldsymbol{d} u \boldsymbol{d} \tau$

$\mathbf{D}_{\mathbf{x}^{[i]} \mathbf{x}^{[j]}}(t, f)=\iint \phi(t-u, \tau) \mathbf{x}^{[i]}\left(u+\frac{\tau}{2}\right)\left(\mathbf{x}^{[j]}\left(u-\frac{\tau}{2}\right)\right)^{H} e^{-j 2 \pi f \tau} \boldsymbol{d} u \boldsymbol{d} \tau$

Spreading Formula (9) in accordance with the horizontal and vertical component, we can get the following formula

$$
\begin{aligned}
& \mathbf{x}(t)=\left[\begin{array}{l}
\mathbf{x}^{[v]}(t) \\
\mathbf{x}^{[h]}(t)
\end{array}\right]=\left[\begin{array}{cc}
\mathbf{A}^{[v]}(\mathbf{\Phi}) & 0 \\
0 & \mathbf{A}^{[h]}(\boldsymbol{\Phi})
\end{array}\right]\left[\begin{array}{l}
\mathbf{s}^{[v]}(t) \\
\mathbf{s}^{[h]}(t)
\end{array}\right]+\left[\begin{array}{l}
\mathbf{n}^{[[]}(t) \\
\mathbf{n}^{[h]}(t)
\end{array}\right] \\
& =\left[\begin{array}{cc}
\mathbf{A}^{[\eta]}(\boldsymbol{\Phi}) & 0 \\
0 & \mathbf{A}^{[h]}(\boldsymbol{\Phi})
\end{array}\right]\left[\begin{array}{l}
\mathbf{Q}^{[p]} \\
\mathbf{Q}^{[h]}
\end{array}\right] \mathbf{s}(t)+\left[\begin{array}{l}
\mathbf{n}^{[[]]}(t) \\
\mathbf{n}^{[h]}(t)
\end{array}\right]=\mathbf{B}(\mathbf{\Phi}) \mathbf{Q} \mathbf{s}(t)+\mathbf{n}(t)
\end{aligned}
$$

It is clear that the dual polarized orthogonal dipole element has more available information than the unipolar element. After theoretical analysis, we can combine the spatial, polarized, and time-frequency domain information of the signal received by the polarization sensitive array. Thus, the spatial polarization timefrequency distribution (SPTFD) of the received data $\mathbf{x}(t)$ can be expressed as

$$
\mathbf{D}_{\mathbf{x x}}(t, f)=\iint \phi(t-u, \tau) \mathbf{x}\left(u+\frac{\tau}{2}\right) \mathbf{x}^{H}\left(u-\frac{\tau}{2}\right) e^{-j 2 \pi f \tau} \boldsymbol{d} u \boldsymbol{d} \tau
$$

\section{Spatial polarimetric time-frequency DOA estimation algorithm}

3.1 Polarimetric time-frequency MUSIC

In [3], it has been proved that the structure of spatial time-frequency distribution matrix is similar to that of the traditional array covariance matrix. Therefore, the STFD matrix can be applied to the subspace class algorithm instead of the covariance matrix in the MUSIC algorithm, called time-frequency MUSIC algorithm.

Similar to the time-frequency MUSIC algorithm, the polarimetric time-frequency MUSIC algorithm, which integrated polarization information, takes the polarization sensitive array for the model and then do time-frequency transform of the received signal shown in Eq. (2) which can obtain the SPTFD matrix. The SPTFD matrix of the corresponding signal can be obtained by sampling each signal along its time-frequency ridge, and the corresponding noise subspace can be obtained by characteristic decomposition.

The following summarizes specific steps of the polarimetric time-frequency MUSIC (PTF-MUSIC) algorithm:

(1)Performing pseudo-Wigner-Ville time-frequency transform to the polarization data received on the first receive channel and estimating the instantaneous frequencies and the frequency modulation slopes of the incoming wave signals in their timefrequency domain.

(2) Using the estimated signal parameters to select points in the time-frequency domain, the different frequency parameters of the signal were selected on their respective time-frequency ridge points to construct their own spatial polarimetric time-frequency distribution matrix.

(3) The eigenvalue decomposition, construction of the noise subspace, and construction of the spatial spectrum are carried out on the constructed spatial polarimetric time-frequency distribution matrix, respectively.

\subsection{Polarimetric time-frequency ESPRIT}

Based on the previous study of MUSIC algorithm and ESPRIT algorithm, we know that they both belong to subspace class algorithm, so similar to MUSIC algorithm, ESPRIT algorithm can also be combined with time-frequency analysis integrated polarization information, which is polarimetric timefrequency ESPRIT (PTF-ESPRIT) algorithm. The biggest advantage of the MUSIC algorithm is the high accuracy of estimation, while the ESPRIT algorithm 
has the biggest advantage of fast calculation. Therefore, the PTF-ESPRIT algorithm should have a faster calculation speed than PTF-MUSIC algorithm in theory. The TLS-ESPRIT algorithm is used in this section.

The principle of PTF-ESPRIT algorithm is similar to the PTF-MUSIC algorithm. Its algorithm flow is as follows:

(1)Performing pseudo-Wigner-Ville time-frequency transform to the polarization data received on the first receive channel and estimating the instantaneous frequencies and the frequency modulation slopes of the incoming wave signals in their timefrequency domain.

(2) Using the estimated signal parameters to select points in the time-frequency domain, for the different frequency parameters of the signal were selected on their respective time-frequency ridge points to construct their own SPTFD matrix.

(3) The covariance matrices $\mathbf{R}_{\mathbf{1 1}}$ and $\mathbf{R}_{\mathbf{2 2}}$ are obtained by the SPTFD matrix.

(4) The covariance matrices are decomposed and two signal subspaces $\mathbf{E}_{\mathbf{1}}$ and $\mathbf{E}_{\mathbf{2}}$ are obtained, then combine them into a new matrix $\overline{\mathbf{E}}$ $=\left[\begin{array}{ll}\mathbf{E}_{1} & \mathbf{E}_{2}\end{array}\right]$.

(5)Construct the matrix $\overline{\mathbf{E}}^{H} \overline{\mathbf{E}}$ and characterize it, we can get the feature matrixE, $\mathbf{E}$ can be divided into four $P \times P$ dimensional matrix.

(6) Calculate $\boldsymbol{\Psi}_{\mathrm{TLS}}=-\mathbf{E}_{\mathbf{1 2}} \mathbf{E}_{\mathbf{2}}^{-1}$ and characterize it, we can get $P$ eigenvalues. And the wave direction information of signals can be obtained according to the formula $k=2 \pi d \sin \theta_{k} / \lambda$.

\subsection{Improved algorithm}

We know that the estimation accuracy of the PTFMUSIC algorithm is very high, but because of its need for a larger angle range for peak search, coupled with the complexity of the time-frequency transform, it takes a long time to get the DOA estimation of the incoming wave signals. The PTF-ESPRIT algorithm does not require spectral peak search, so it takes much less time than the PTF-MUSIC algorithm, but its estimation accuracy is not so good. Therefore, an improved algorithm is proposed in this paper, and the advantages of the two algorithms are comprehensively used to estimate the DOA.

The principle of the improved algorithm is as follows. Firstly, the pseudo-Wigner-Ville time-frequency transform of the first channel polarization data received by the array is used to obtain its polarization time-frequency distribution figure and then analyze and extract the SPTFD matrix on the time-frequency ridge of each signal, and use it to replace the traditional covariance matrix; the DOA of signal will be estimated by TLS-ESPRIT algorithm roughly. The arrival angles of the different signals are locked in a range. In this range, the MUSIC algorithm is used to estimate the DOA accurately. This will not only save time but also improve the estimation accuracy; the algorithm flow is shown in Fig. 2.

As shown in Fig. 2, the specific process of the improved algorithm is as follows:

(1)Firstly, the pseudo-Wigner-Ville time-frequency transform is performed on the polarization data received by the first channel of the array, and the instantaneous frequency and frequency modulation slope of the incoming wave signal are estimated in the time-frequency domain.

(2)With the estimated signal parameters to select points in the time-frequency domain, for signals of different frequency parameters, the points on respective time-frequency ridge were selected to construct their own SPTFD matrix.

(3) The DOA estimation of the time-frequency ESPRIT algorithm is used to obtain a general range of DOAs for each signal.

(4) The PTF-MUSIC algorithm is carried out in the DOA range obtained in the previous step, and the spatial spectrum is constructed to obtain the accurate DOA estimation value.

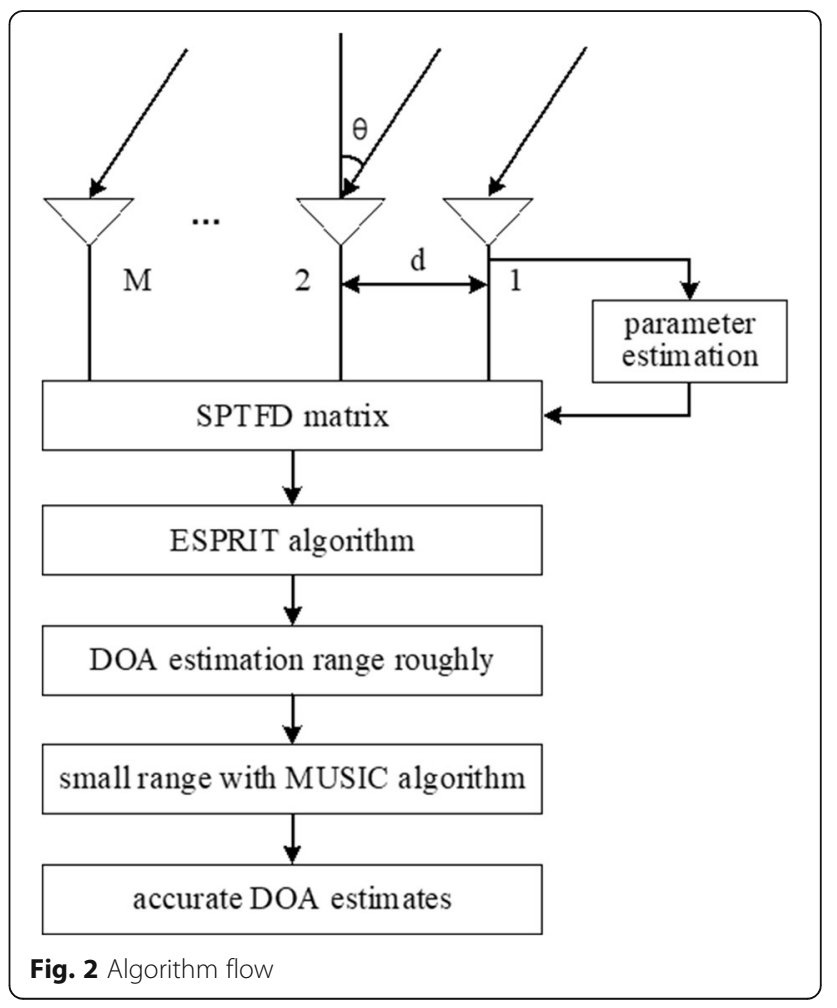




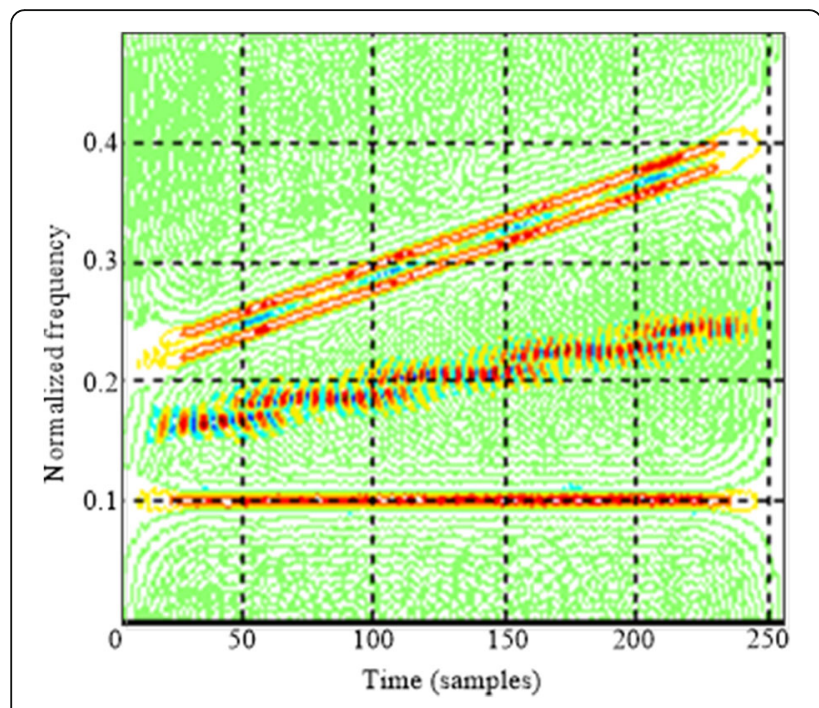

Fig. 3 WD time-frequency transform of signals

\section{Simulation results}

\subsection{Polarimetric time-frequency MUSIC}

\subsubsection{Experiment 1: TF MUSIC vs PTF MUSIC}

Assuming a 4-element polarized sensitive array consists of dual polarized orthogonal dipoles and three linear frequency modulation signals incident on it, the normalized frequencies of the three signals are 0.2 to $0.4,0.22$ to 0.42 , and 0.1 ; DOAs are $-3^{\circ}, 3^{\circ}$, and $9^{\circ}$; polarized angles are $45^{\circ}, 45^{\circ}$, and $20^{\circ}$; and polarized phase angles are $0^{\circ}$, $180^{\circ}$, and $0^{\circ}$, respectively. Take the data length of 256 , the signal to noise ratio of $13 \mathrm{~dB}$, assuming that the number of signals and their polarized parameters are known. In this experiment, DOA estimation of signal 1 and signal 2 are carried out by time-frequency MUSIC and polarized time-frequency MUSIC algorithm. Each algorithm performs three independent experiments, analyzes its time-frequency transform, and draws spatial spectrum. First of all, the WVD time-frequency transform of the received data is shown in Fig. 3.

By the polarized state of the signals, we can see that $s_{1}$ (source 1) and $s_{2}$ (source 2) satisfies the conditions of electromagnetic vector quadrature. So cross-term between the two signals is suppressed when the timefrequency distribution of the two directional components is taken into account. This helps us select the time-frequency points of signals more accurately.

Figure 4 is the spatial spectrum obtained by timefrequency MUSIC algorithm and polarized timefrequency MUSIC algorithm to signal 1 and signal 2, each algorithm has been done three independent experiments.

From Fig. 4, we can see that signal 1 and signal 2 cannot be distinguished correctly by the MUSIC spatial spectrum obtained by the time-frequency information of the unipolar component because their time-frequency points are too near and there are very serious crossterm, resulting in fuzzy of their time-frequency information. However, the PTF MUSIC algorithm can distinguish the two signals very easily because the two signals are orthogonal to the electromagnetic information, so that their time-frequency information is very clear and their time-frequency points can be easily extracted, and the resulting spectral peak is very sharp.

\subsubsection{Experiment 2: estimated accuracy of TF MUSIC vs PTF MUSIC}

Assuming a 4-element polarized sensitive array consists of dual polarized orthogonal dipoles and two LFM signal incidents on it, the normalized frequencies of the two signals are 0.2 to 0.4 and 0.1 , DOAs are $-3^{\circ}$ and $9^{\circ}$, polarized angles are $45^{\circ}$ and $20^{\circ}$, and polarized phase angles are $0^{\circ}$ and $180^{\circ}$, respectively. Taking the data length of 256 , assuming the number of signals and their polarized parameters are known, the SNR increases from - 10 to
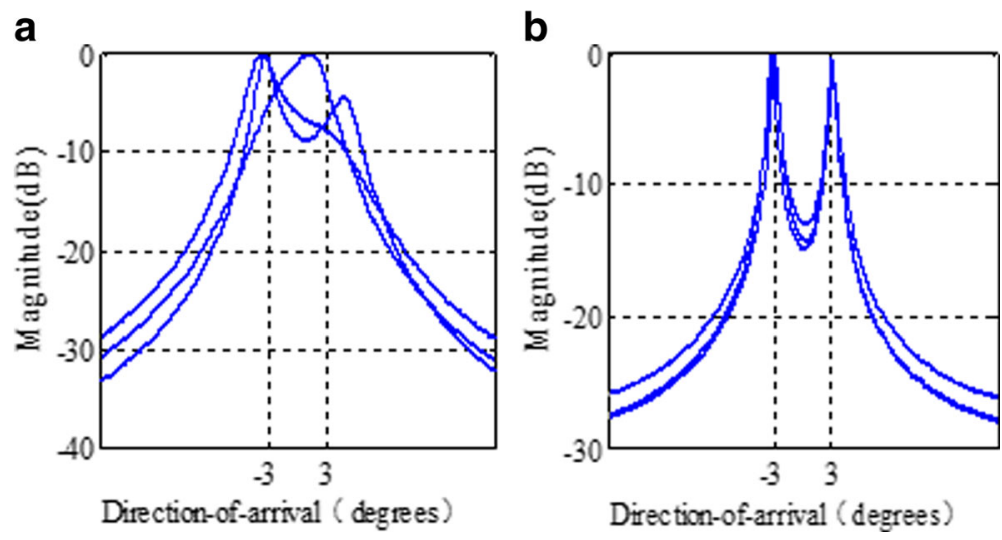

Fig. 4 Spatial spectrum: a Spatial spectrum of TF MUSIC algorithm. b Spatial spectrum of PTF MUSIC algorithm 


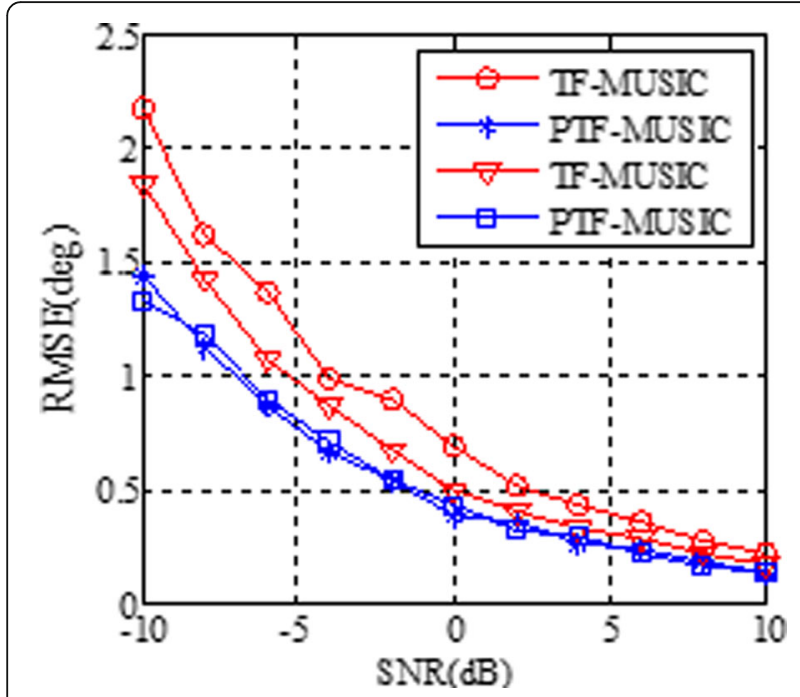

Fig. 5 RMSE of TF MUSIC and PTF MUSIC

$10 \mathrm{~dB}$ with an increase of $2 \mathrm{~dB}$. The Monte Carlo experiments were carried out 200 times to calculate the RMSE of each DOA estimation by using the TF MUSIC algorithm and PTF MUSIC algorithm, respectively; the results are shown in Fig. 5.

As can be seen from Fig. 5, the RMSE of the two DOA estimates both decreases with the increase of the SNR between two algorithms, which means that the accuracy of the DOA estimation is getting higher and higher. Overall, the accuracy of the DOA estimate is improved after taking full advantage of the polarized information of the electromagnetic signals, especially in the case of low SNR.

\subsection{Polarimetric time-frequency ESPRIT}

\subsubsection{Experiment 3: TF ESPRIT vs PTF ESPRIT}

Assuming a 4-element polarized sensitive array consists of dual polarized orthogonal dipoles and three LFM signal incidents on it, the signal parameters are the same as in experiment 1. Take the data length of 256, and the SNR of $13 \mathrm{~dB}$, assuming that the number of signals and their polarized parameters are known. In this experiment, the TF ESPRIT and PTF ESPRIT algorithm are used to estimate DOAs of signal 1 and signal 2 and each algorithm performs 100 independent Monte Carlo experiments. The average values of DOAs are calculated respectively, and the results are shown in Table 1.

Table 1 The average value of the DOA estimates of two algorithms

\begin{tabular}{lll}
\hline Signal & TF-ESPRIT $\left(^{\circ}\right)$ & PTF-ESPRIT $\left({ }^{\circ}\right)$ \\
\hline Signal 1 & -26.8481 & -3.0058 \\
Signal 2 & 1.6727 & 3.0129 \\
\hline
\end{tabular}

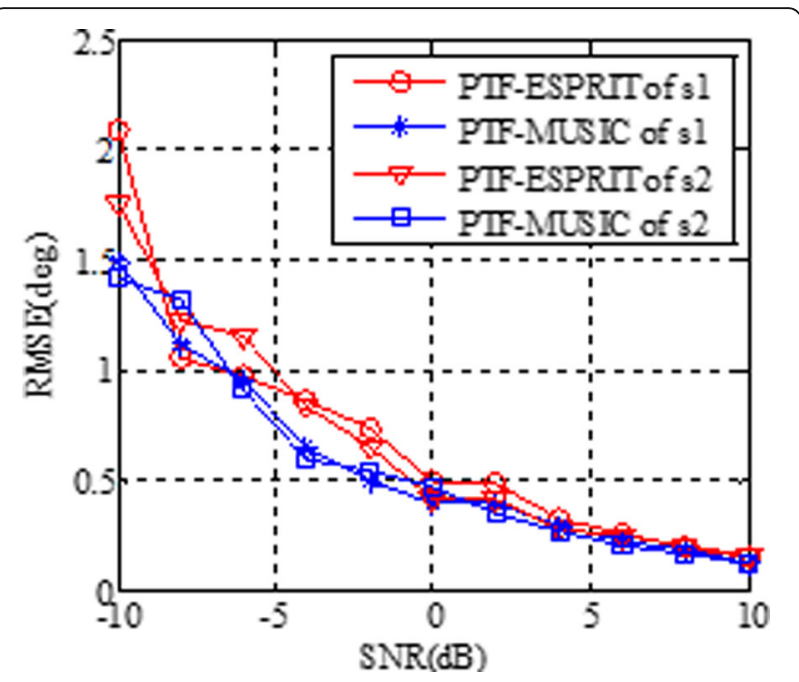

Fig. 6 RMSE of two PTF DOA estimation algorithms

From Table 1, we know that since the time-frequency information of signal 1 and signal 2 is very similar and there are cross terms, the TF ESPRIT algorithm cannot distinguish them easily. But because the signals have different polarized information, the PTF ESPRIT algorithm can distinguish the two signals correctly.

\subsubsection{Experiment 4: PTF ESPRIT vs PTF MUSIC}

Assuming a 4-element polarized sensitive array consists of dual polarized orthogonal dipoles and two LFM signal incidents on it, the signal parameters are the same as in experiment 2. Take the data length of 256 , and the noise is Gaussian white noise uniformly distributed, assuming that the number of signals and their polarized parameters are known. The SNR increases from -10 to $10 \mathrm{~dB}$ with an increase of $2 \mathrm{~dB}$, the Monte Carlo experiments were carried out 100 times to calculate the RMSE of each DOA estimation by using the PTF MUSIC and PTF ESPRIT algorithm, respectively; the results are shown in Fig. 6. In addition, the time to calculate the time-frequency transformation and the two algorithms are shown in Table 2.

It can be seen from Fig. 6 that the RMSE of the DOA estimates of the two signals estimated by the PTF MUSIC and PTF ESPRIT algorithm both decreases with the increase of the SNR, which means that the accuracy of DOA estimation is also getting higher and higher. Comparing the two algorithms, it can be seen that the

Table 2 The time of algorithms

\begin{tabular}{lll}
\hline Algorithm & Time $(\mathrm{s})$ & Time of algorithm (s) \\
\hline TF transformation & 434.5292 & 434.5292 \\
PTF ESPRIT & 453.4780 & 18.95 \\
PTF MUSIC & 540.3157 & 105.7865 \\
\hline
\end{tabular}


estimated accuracy of the PTF MUSIC algorithm is higher than PTF ESPRIT algorithm under the same conditions, especially in the case of low SNR.

Table 2 describes the computer consumed times of two algorithms, the computer configuration are as follows: CPU is Intel Pentium E5200 (Dual core), memory is $4 \mathrm{G}$, and $\mathrm{OS}$ is Window10 platform. It can be seen from Table 2 that the TF transformations occupy a considerable amount of time; in addition, the PTF ESPRIT algorithm only needs $18.95 \mathrm{~s}$, while the PTF MUSIC algorithm needs $105.7865 \mathrm{~s}$, about 6 times of the PTF ESPRIT algorithm. In this experiment, the spectral peak search is only between $-20^{\circ}$ to $20^{\circ}$, if the DOA of signal incident is from a wide range of angles, the time consuming of PTF MUSIC algorithm will be as several dozen times as PTF ESPRIT algorithm.

\subsection{Improved algorithm}

Assuming a 4-element polarized sensitive array consists of dual polarized orthogonal dipoles and two LFM signal incidents on it, the snapshot numbers are 256 and the normalized start and end frequencies of the two signals are $0.2 \sim 0.4$ and $0.1 \sim 0.1$. The SNR increases from 0 to $10 \mathrm{~dB}$ with an increase of $2 \mathrm{~dB}$, use the PTF MUSIC, PTF ESPRIT and improved algorithm to estimate the DOAs of incident signals, respectively. Each algorithm performs 100 independent experiments to calculate the RMSE, the results are shown in Fig. 7.

It can be seen from Fig. 7 that the improved algorithm and the PTF MUSIC algorithm have similar estimated accuracy, which are higher than that of the PTF ESPRIT algorithm.

Experiment 6: the run time of the improved algorithm

The experimental condition is similar to those of experiment 5 , except that the SNR is fixed at $10 \mathrm{~dB}$. This experiment calculates the run time required for 100 times of Monte Carlo experiments for each algorithm, as shown in Table 3.
Table 3 The time of algorithms

\begin{tabular}{lll}
\hline Algorithm & Time $(\mathrm{s})$ & Time of algorithm $(\mathrm{s})$ \\
\hline PTF transformation & 43.8828 & 43.8828 \\
PTF MUSIC & 138.7203 & 94.8195 \\
PTF ESPRIT & 47.1496 & 3.2668 \\
Improved algorithm & 48.8823 & 4.9995 \\
\hline
\end{tabular}

As can be seen from Table 3, the time spent of PTF MUSIC and improved PTF DOA estimation algorithms are 138.7203 and $48.8823 \mathrm{~s}$, respectively. Yet timefrequency transform needs to be carried out in each experiment and the time of 100 times of time-frequency transform is $43.8828 \mathrm{~s}$, which means that the PTF MUSIC algorithm and improved PTF DOA estimation algorithm spend 94.8195 and $4.9995 \mathrm{~s}$, respectively. Meanwhile, the PTF ESPRIT algorithm spends about $3.2668 \mathrm{~s}$, so the time required for PTF MUSIC algorithm is about 19 times of the time for the improved PTF DOA estimation algorithm, and is about 29 times of the time used for the PTF ESPRIT algorithm under the same circumstances. This experiment shows that the improved PTF DOA estimation algorithm optimizes the time-consuming problem to a large extent under the condition of guaranteeing estimation accuracy.

\section{Conclusions}

This paper focuses on the principle of polarized timefrequency MUSIC and ESPRIT algorithms, and the improved algorithm is proposed based on the advantages and disadvantages of both of them. The improved algorithm makes full use of the advantages of the small calculation of ESPRIT algorithm and the high accuracy of MUSIC algorithm, which makes the algorithm more practical and the computer simulation also proves its performance. However, there are still some places need to be improved, such as the pseudo-WV distribution is suitable for the processing of LFM signals, but its kernel
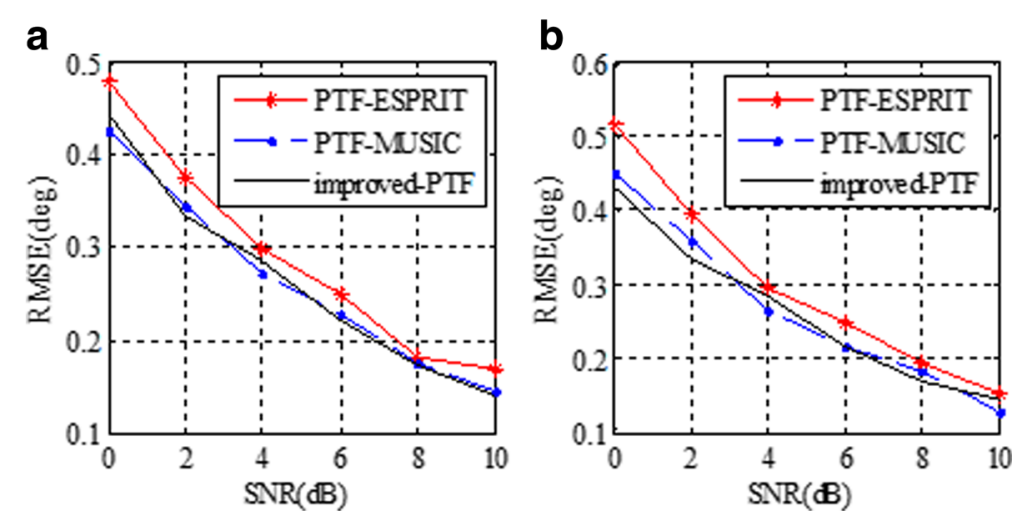

Fig. 7 RMSE of two signals: a RMSE of signal 1. b RMSE of signal 2 
function cannot adapt to different forms of signals, and it also has cross terms to a certain extent. Therefore, it is worth considering how to design a kernel function that changes adaptively with the signal forms and does not have cross terms.

\section{Abbreviations}

DOA: Direction of Arrival; ESPRIT: Estimating Signal Parameters Viarotational Invariance Techniques; HFSWR: High Frequency Surface Wave Radar; LFM: Linear Frequency Modulation; MUSIC: Multiple Signal Classification; SNR: Signal-to-Noise Ratio; SPTFD: Spatial Polarization Time-Frequency Distribution

\section{Acknowledgements}

This project is sponsored by the National Marine Technology Program for Public Welfare (no. 201505002), the National Natural Science Foundation of China (nos. 61571157, 61401118, and 61371100), and the Subject Guide Fund of Harbin Institute of Technology at Weihai (no. WH20150111).

\section{Funding}

The National Marine Technology Program for Public Welfare (no. 201505002) is supporting the data acquisition devices and materials, the National Natural Science Foundations of China (nos. 61571157, 61401118, and 61371100) are supporting the simulations, and the Subject Guide Fund of Harbin Institute of Technology at Weihai (no. WH20150111) is supporting the data analyses.

\section{Availability of data and materials}

Not applicable.

\section{Authors' contributions}

$A L$ and $B L$ conceived and designed the experiments, FL performed the experiments, QL and XS contributed simulation tools, and AL and BL wrote the paper. All authors have read and approved the final manuscript.

\section{Authors' information}

Not applicable.

\section{Competing interests}

The authors declare that they have no competing interests.

\section{Publisher's Note}

Springer Nature remains neutral with regard to jurisdictional claims in published maps and institutional affiliations.

\section{Author details}

${ }^{1}$ School of Information and Electrical Engineering, Harbin Institute of Technology (Weihai), Weihai, China. ${ }^{2}$ Shanghai Radio Equipment Research Institute, Shanghai, China.

Received: 27 November 2017 Accepted: 14 February 2018

Published online: 05 March 2018

\section{References}

1. A Belouchrani, M Amin, Blind source separation based on time-frequency signal representation. IEEE Trans. SP 46(11), 2888-2898 (1998)

2. A Belouchrani, M Amin, Time-frequency MUSIC. IEEE Signal Process. Lett. 6(5), 109-110 (1999)

3. W Sharif, Y Chakhchoukh, AM Zoubir, Direction-of-arrival estimation of FM sources based on robust spatial time-frequency distribution matrices. IEEE Stat. Signal Process. Workshop (SSP), 537-540 (2011)

4. Y Zhang, W Mu, M Amin, Time-frequency maximum likelihood methods for direction finding. Franklin Inst. 337(4), 483-497 (2000)

5. KT Wong, MD Zoltowski, Uni-vector-sensor ESPRIT for multisource azimuth, elevation, and polarization estimation. IEEE Trans. Antennas Propagation 45(10), 1467-1474 (1997)

6. A Nehorai, P Tichavsky, Cross-product algorithms for source tracking using an EM vector sensor. IEEE Trans. SP 47(10), 2863-2867 (1999)
7. L Sun, G Ou, Y Lu, Vector sensor cross-product for direction of arrival estimation. IEEE 2nd International Congress on Image and Signal Process, $1-5(2009)$

8. KT Wong, MD Zoltowski, Closed-form direction finding and polarization estimation with arbitrarily spaced electromagnetic vector-sensors at unknown locations. IEEE Trans. Antennas Propagation 48(5), 671-681 (2000)

9. Xu Y, Liu Z. Regularized ESPRIT-based direction finding and polarization estimation with one electromagnetic vector sensor. IEEE 7th International Conference on Signal Processing, 2004: 399-402.

10. X Yuan, Estimating the DOA and the polarization of a polynomialphase signal using a single polarized vector-sensor. IEEE Trans. SP 60(3), 1270-1282 (2012)

11. E Ferrara, T Parks, Direction finding with an array of antennas having diverse polarizations. IEEE Trans. Antennas Propag. 31(2), 231-236 (1983)

12. M Costa, A Richter, $V$ Koivunen, DOA and polarization estimation for arbitrary array configurations. IEEE Trans. SP 60(5), 2330-2343 (2012)

13. X Zhang, C Chen, J Li, et al., Blind DOA and polarization estimation for polarization-sensitive array using dimension reduction MUSIC. Multidim. Syst. Sign. Process. 25(1), 67-82 (2014)

14. L Liu, Y Jiang, L Wan, et al., Beamforming of joint polarization-space matched filtering for conformal array. Sci. World J. 2013(2), 1653-1656 (2013)

15. MR Bell, N Oppermann, A Crai, et al., Improved CLEAN reconstructions for rotation measure synthesis with maximum likelihood estimation. Astron. Astrophys. 551(3), 309-314 (2012)

16. $Y X u, Z$ Liu, Polarimetric angular smoothing algorithm for an electromagnetic vector-sensor array. IET Radar Sonar Navigation 1(3), 230-240 (2007)

\section{Submit your manuscript to a SpringerOpen ${ }^{\circ}$ journal and benefit from:}

- Convenient online submission

- Rigorous peer review

- Open access: articles freely available online

- High visibility within the field

- Retaining the copyright to your article

Submit your next manuscript at $>$ springeropen.com 